\title{
Editorial
}

\section{Resistencias y emociones del activismo en contextos represivos, autoritarios o violentos. Una introducción}

\author{
Alice Poma \\ Universidad Nacional Autónoma de México, Ciudad de México, México \\ Email: alicepoma@gmail.com \\ Juan Pablo Paredes \\ Universidad de los Lagos, Santiago, Chile. \\ Email: juan.paredes2@ulagos.cl \\ Tommaso Gravante \\ Universidad Nacional Autónoma de México, Ciudad de México, México \\ Email: t.gravante@gmail.com
}

\section{Las emociones como arena de la lucha política}

Desde los años noventa del siglo pasado el campo de estudio de los movimientos sociales se ha enriquecido gracias a la incorporación de la dimensión emocional como factor explicativo de las luchas y las protestas sociales (véase Poma y Gravante 2017a, para un estado del arte actualizado en español).Las emociones han sido incorporadas al estudio de la protesta para analizar distintas etapas y procesos que caracterizan los movimientos sociales. Como sugiere Flam (2005) la mayoría de los trabajos que incorporan las emociones al estudio de la protesta se han centrado en los procesos de carácter micro-político, especialmente en el rol de las emociones en la movilización (mobilizing emotions) y en cómo los movimientos sociales transforman y gestionan los sentimientos de sus miembros. En cuanto al papel de las emociones en la motivación a la acción, ya mucha literatura ha confirmado que las emociones cuentan con una gran capacidad explicativa tanto a nivel individual como colectivo. A nivel individual las emociones motivan el activismo y permiten entender por qué los individuos deciden involucrarse en la acción, incluso cuando los costes de la movilización puedan superar sus beneficios, mientras que a nivel colectivo crearían el ambiente favorable para el desarrollo de la movilización. Por otro lado, a lo largo de estas décadas, la incorporación de la dimensión emocional ha permitido ayudar a explicar el origen, el desarrollo y el éxito o no, de un movimiento social, pero también ha permitido comprender las tensiones, divisiones y problemas internos de los grupos, lo que pasa cuando los movimientos terminan; participando de manera relevante en los procesos vinculados al desarrollo de estas experiencias, en la emergencia o fortalecimiento de la identidad colectiva, la construcción de un marco de injusticia, la construcción de una amenaza común y del shock moral, así como los impactos de la protesta en su dimensión cultural y biográfica.

El principal enfoque basal sobre las emociones de todas estas múltiples investigaciones ha sido que las emociones son constructos culturales y sociales. Un enfoque que tiene su origen en la propuesta teórica de la sociológica estadounidense Arlie Hochschild $(1979,1983)$ que desde finales de los años setenta desarrolla estudios empíricos con estudiantes y trabajadores para analizar el trabajo emocional que estas personas hacen en su vida cotidiana y su lugar de trabajo, demostrando que en cada cultura existen reglas del sentir que los seres humanos seguimos para encajar en la sociedad. A diferencia de la psicología que considera las emociones cómo estados internos individuales y biológicos, actuando sobre el individuo para la resolución de problemas, 
Hochschild, por un lado, considera a las emociones como una construcción sociocultural y por lo tanto cambiante en función del contexto social y de la temporalidad histórica, superando de esta forma la visión organicista y universal de las emociones. Por otro lado, la misma autora considera al individuo como un ser consciente y activo con respecto a sus emociones, es decir, a diferencia de Freud, para Hochschild las personas son conscientes de sus propias emociones. En tanto, a diferencia de Goffman, las personas no solamente son capaces de hacer un actuación superficial -superficial acting-manifestando de esta manera las emociones más oportunas y acordes con la situación, más bien pueden hacer una interpretación profunda de sus propios sentimientos -deep acting- evocando, manejando o canalizando una determinada emoción para adecuarse o desafiar las reglas del sentir de su propia sociedad. De esta forma la propuesta de Hochschild va a vincular las situaciones sociales y la macro estructura, por un lado, y la personalidad por el otro. De lo anterior se sigue que las personas se vuelven sujetos activos con respecto a sus emociones, pueden pensar sobre lo que sienten o utilizar determinadas emociones para evocar otras.

El legado de Arlie Hochschild $(1975,1979,1983)$ mostró que la evocación de determinadas emociones y las reglas del sentir pueden convertirse en "objetos de la lucha política” (Hochschild, 2008, p. 149), y tal como señalamos anteriormente, su aporte fue recuperado después de dos décadas por los estudiosos de los movimientos sociales, cuya principal aportación analítica y metodológica ha sido la de considerar y definir a las emociones como variables explicativas de la acción colectiva contenciosa y de sus múltiples procesos, al punto que hoy es difícil entregar explicaciones de procesos contenciosos sin considerar seriamente el papel de las emociones en ellos.

\section{Emociones y activismo de alto riesgo}

De lo anterior, superando la visión universal y organicista, se desprende que las emociones son parte de la cultura, de los contextos socio históricos y de la biografía de las personas (Jasper, 1997). Por lo tanto, en diferentes contextos socioculturales se pueden obtener resultados distintos a pesar de la misma pregunta de investigación o a pesar de considerar una misma emoción como variable explicativa. Por ejemplo, el miedo a la represión puede paralizar como unir a los miembros de un colectivo (Poma y Gravante, 2017b y 2018a), y otras emociones como la impotencia, la desesperanza, el agobio, la frustración, pueden llevar al abandono de la lucha cuando se acompañan con la resignación, aunque también pueden ser sobrellevadas por los activistas y no claudicar en su lucha.

Entre otros aspectos, el análisis de la dimensión emocional se ha mostrado útil en el análisis de movimientos o de activismo de alto riesgo (McAdam 1986, Goodwin y Pfaff, 2001), es decir, experiencias que se desarrollan en contextos de alta represión y violencia, por ejemplo conflictos armados, regímenes políticos autoritarios o totalitarios, en los cuales los activistas y participantes involucrados en resistencias, protestas y movimientos sociales, viven y enfrentan situaciones de inseguridad y amenazas en sus actividades políticas cotidianas. En estos contextos, otras teorías de los movimientos sociales como la movilización de los recursos o de las oportunidades políticas, basadas en la acción racional y aplicadas en su mayoría en contextos democráticos liberales, no han logrado explicar dinámicas referidas al porqué los activistas seguían enfrentando y oponiéndose a situaciones de injusticia, a pesar del miedo a la alta represión; o cómo podían los grupos y colectivos evitar el agotamiento y los costes de la movilización; o cuáles fueron los beneficios de la movilización en contextos represivos sin posibilidad de apertura política.

Sin duda, los distintos grados de represión que pueden caracterizar el activismo de alto riesgo, como la desaparición forzada de activistas, el asesinato de luchadores sociales, las amenazas de muerte, el acoso, la violencia física y psicológica por parte de policías, ejércitos, grupos criminales y grupos opositores, entre otros, tienen un fuerte impacto en las actividades políticas de los colectivos y grupos sociales, al punto que deben ser manejadas por los miembros de estos grupos. Sin embargo, las emociones que emergen en tales situaciones influyen siempre de forma distinta y no mecánica.

Una de las primeras investigaciones que utilizó este enfoque en un contexto represivo fue la investigación de Helena Flam en 1998. La socióloga de las emociones polaca hace un esfuerzo de macro sociología 
relacionando como determinadas emociones, como por ejemplo el miedo, han permitido la estabilidad en un largo periodo de tiempo del sistema soviético en dos países como Polonia y Alemania del Este antes de 1989. Con más de ochenta entrevistas biográficas y treinta entrevistas en profundidad, Flam analiza el recorrido de militantes del partido comunista y de disidentes de los dos países (más un tercer grupo de personas no involucradas políticamente como grupo de contraste), comprobando como el miedo cambia radicalmente en función del contexto histórico. Por ejemplo, en la era post-Stalin, las personas ya no tenían miedo por sus vidas ni de sufrir de tortura como antes, más bien, tenían miedo por sus oportunidades de vidas, reflejando de esta forma el nuevo rol del partido comunista capaz de controlar y normar las oportunidades de vida de la población. La forma en que se manejaba tal ansiedad con respecto a las oportunidades de vida, para si y sus familiares, fue un factor determinante y se diferenciaba entre los miembros del partido comunista y los disidentes, así como entre los grupos a los cuales cada uno pertenecía y a su contraparte al otro lado de la frontera. Como en otros contextos altamente autoritarios, el manejo emocional de determinadas emociones- por ejemplo, la canalización del miedo en esperanza, de la tristeza en alegría o el manejo de la ansiedad-, resultó central para la vida cotidiana de las personas, la sobrevivencia de los grupos y el desarrollo de sus actividades normales. Quedándonos en la misma región política, años más tarde Colin Barker (2001) analizó la emergencia del sindicato no gubernamental polaco Solidarno?? en 1980 y mostró cómo determinadas emociones como la hermandad, la alegría, el orgullo y los procesos de manejo emocional del miedo y de la ansiedad, permitieron al naciente sindicado polaco fortalecerse y hacer frente al clima de represión que caracterizaba a Polonia en aquella época. Emociones como la hermandad, la confianza, la lealtad, el compartir que actuaban por una causa justa y que la victoria era inevitable, entre otras, permite construir relaciones sociales íntimas que fortalecen los movimientos en contextos de altos riesgos. Como destacan Goodwin y Pfaff (2001), comparando el movimiento para los derechos civiles en EE.UU entre los 50s y los 60s y el movimiento para los derechos civiles o movimiento cívico en la Alemania del Este en los 80, estas estructuras, redes informales y vínculos afectivos han sido importantes porque han animado los activista en superar las adversidades.

Una de las preguntas centrales en el estudio de los movimientos sociales -¿̇porqué la gente se moviliza?adquiere particular relevancia en contextos de alto riesgo, de guerrillas armadas o de guerra, donde la violencia política y represiva es común. Si Helena Flam indicó cómo el manejo emocional de determinadas emociones resulta clave en el desarrollo de las actividades de los grupos políticos (pro-régimen o disidentes), otras investigaciones hacen hincapié en cómo y porqué las personas se involucran en organizaciones clandestinas armadas o siguen apoyando grupos armados a pesar de poner en riesgo su propia vida. Los fuertes sentimientos de solidaridad, lealtad y hermandad representan elementos claves en la participación de jóvenes en los grupos guerrilleros, como las FARC en Colombia (Bolivar, 2006; Otero, 2006), además de los beneficios emocionales como la admiración y respeto, el hacerse respetar y la dotación de sentido. Como señaló Donatella della Porta (1998), aquellos que ingresan en los grupos armados suelen romper con sus antiguos lazos sociales como la familia y los amigos, pues el componente emocional permite una segunda socialización-casi tan fuerte como la primaria-, capaz de dar significado a su proceso de radicalización.

Otro concepto que el enfoque de las emociones proporciona para comprender a los movimientos sociales es el de beneficios emocionales (Whittier, 2011). Algunas emociones, como el orgullo y la dignidad que las personas sienten al participar en cientos movimientos, o al actuar según sus principios, como que "haciendo la cosa justa”, así como con la esperanza que la situación pueda cambiar, entre otras, se pueden convertir en beneficios emocionales de la participación en las organizaciones clandestinas armadas. Además, representan un elemento crucial para comprender el involucramiento de gente normal y corriente, como los campesinos, en apoyar a estas organizaciones. Elisabeth Wood (2003), desde la dimensión emocional, explica porqué en El Salvador a lo largo de la guerra civil, una parte del campesinado apoyó la insurgencia del Frente Farabumdo Martí para la Liberación Nacional (FMLN). Los campesinos, aunque no participaron en la guerrilla, apoyaron la organización guerrillera a través de comida y otras necesidades logísticas. Para la autora una de las motivaciones de este apoyo, a pesar de los riesgos que esto presuponía, se encuentra en los beneficios morales y afectivos que los campesinos recibían a través de su participación, aun cuando era evidente la derrota del FMLN. A través de su apoyo al FMLN, los campesinos desarrollaban un sentimiento de orgullo y dignidad que se contraponía a las emociones, como la rabia o el ultraje, experimentadas al haber sido explotados. De este modo el ultraje y el orgullo, junto con las otras razones convencionales como el acceso a las tierras libre, impulsaron la participación de los campesinos en la insurgencia a pesar de los altos riesgos e incertidumbre. 
Si las emociones morales (véase Jasper 2018, para la categorización completa) representan un elemento central para la comprensión del activismo de alto riesgo, han sido identificadas también otra tipología de emociones como los estados de ánimos, entre los que se encuentran la resignación o la desesperación, que pueden llevar a lo que Jasper (2018, p.85) llama el nothing-left-to-lose effect. Ese proceso explica cómo los sujetos al sentir que no tienen nada que perder, pueden sobrellevar el miedo o hacerlo desaparecer, para dejar su lugar a la rabia, la venganza, el orgullo o la dignidad. Uno de los más famosos movimientos que emerge desde un desesperado dolor y un fuerte ultraje moral es el movimiento de las Madres de Plaza de Mayo en Buenos Aires en 1976, en plena dictadura militar argentina. La desesperación junto a la frustración del silencio por parte de las autoridades argentinas, entre otros aspectos, desencadenó el efecto descripto por Jasper, permitiendo la emergencia del movimiento (y la continuidad por más de treinta años) de madres que habían perdido sus hijos y que probablemente se involucraban por primera vez en una actividad política. El nothing-left-to-lose effect, adquiere suma relevancia en sistemas dictatoriales como el caso de Argentina y en situaciones de alto control social, como es el caso de las cárceles especiales donde el único control que queda al prisionero es el manejo de su propio cuerpo y por lo tanto su única forma de protesta puede ser la huelga de hambre llevada hasta las extremas consecuencias, o en situaciones de guerra y ocupaciones militares como es el caso de Palestina. Robert Bryam y Bader Araj (2006) en sus investigaciones encuentran que la mayoría de los suicidas bombas palestinos (entre 2000 y 2005) eran motivados más por un deseo de venganza y represalia, que una motivación estratégica y política (aunque ella se haga presente). Haber perdido a miembros de su familia, sus amigos o algunos de sus seres queridos, como el sufrir las continuas humillaciones por parte de los israelitas o sentir que ni el honor queda, convierte la venganza no solamente es un objetivo necesario, sino en la única alternativa posible, ya que no queda nada por lo que vivir.

El manejo emocional que se hace al canalizar o mitigar emociones como la rabia en otro tipo de emoción (Poma y Gravante, 2018b) resulta central en contextos autoritarios para evitar una mayor represión, el agotamiento del grupo o la impotencia frente a un contendiente más fuerte y violento. En otros casos, los activistas hacen un uso estratégico del trabajo emocional para evocar emociones como la rabia o la culpa. En su investigación sobre el movimiento abolicionista en EE.U., Benjamin Lamb-Books (2016) analiza el uso estratégico de determinadas emociones por parte de los activistas del norte de Estados Unidos que promovían la abolición de la esclavitud racial. En las reuniones públicas, la problematización de la esclavitud racial se realizaba intentando contagiar la audiencia con sentimientos como la rabia y el ultraje, juntos con la amenaza y con estados de ánimos como el optimismo. Es interesante ver como cada grupo abolicionista trabajaba sobre emociones distintas: por ejemplo, los miembros de la Iglesia Evangélica involucrados en el movimiento abolicionista trabajaron más en construir un marco emocional en el cual la esclavitud se enmarcaba como un pecado nacional, una corrupción del poder, los sentidos de culpa, complicidad y ultraje moral. De la misma manera el historiador Zeldin (1979), hace hincapié en la importancia de la rabia en la política francesa desde la Francia revolucionario de 1848 hasta la Segunda Guerra Mundial. La rabia se convierte en estos casos en un indicador de haber sufrido una injusticia, así como las comunidades zapatistas rebeldes en México que reivindican una digna rabia para todas las injusticias sufridas en las poblaciones indígenas a lo largo de los siglos (Poma y Gravante, 2019, p. i-iii).

Por último, hay que considerar que también en el activismo de alto riesgo las manifestaciones de determinadas emociones se vinculan al evidente contexto represivo, por un lado, pero por el otro siguen vinculadas a las reglas del sentir dominantes. Por ejemplo, en grupos religiosos la rabia resulta una emoción que es necesario reprimir o por lo menos no manifestar públicamente. Sin embargo, es interesante ver cómo en determinados contextos que emergen de conflictos sociales violentos como las guerras se pueden introducir nuevas reglas del sentir o cambiar de significado a determinadas emociones. Por ejemplo, la investigación de Venessa Pouvarac (2004) en la ex-Yugoslavia, en particular en Bosnia, muestra como el nuevo paradigma de seguridad internacional centrado en la seguridad ofrecida por las Naciones Unidas, introdujo un acercamiento terapéutico AngloAmericano a los conflictos violentos cuyo objetivo fue despolitizar la rabia. Como muestra la autora, el resultado de este programa fue un incremento del sentido de frustración y alienación de la población local hacia los procesos políticos.

A nivel empírico los resultados de las investigaciones presentadas en estas páginas demuestran cómo las emociones que se generan en experiencias de activismo de alto riesgo y de represión política se convierten en un factor clave para comprender procesos que las teorías basadas en la elección racional no logran explicar. 
Como muestran distintos casos, emociones como el miedo a la represión, la tortura o a perder la vida, representan una constante en determinados contextos sociales. Aunque estas emociones pueden movilizar, también pueden desmovilizar, puesto que es el manejo y la interpretación de lo que los sujetos sienten lo que permite entender su comportamiento, practica y acciones. Conjuntamente con las demás actividades políticas y organizativas, el trabajo reflexivo, individual y colectivo sobre las emociones es clave para que las personas no se desgasten y las luchas no acaben. Manejar el miedo, la impotencia, la soledad, entre otras, es la única manera para determinados grupos para desarrollar sus actividades.

A nivel teórico es necesario todavía un esfuerzo para poder sistematizar la relación que hay entre las emociones sentidas individualmente, la interrelación que ocurren entre ellas y las modalidades de mutación o transformación que experimentan en función de aristas tan relevantes como el contexto represivo, la percepción de la amenaza o del riesgo, el sentimiento de ultraje, entre otros. El nothing-left-to-lose effect propuesto por Jasper, nos permite comprender el pasaje desde un estado de ánimo profundo a una emoción moral en contextos adversos, siendo un primer paso en este camino.

El número 53 de Polis, intenta ser un aporte a la discusión y reflexión a escala regional sobre la relación entre procesos de movilización social y los registros emocionales implicados en ellos. Al poner el foco de atención en el papel que juegan las emociones en el activismo en contextos de riesgo, ya sea por situaciones de violencia política, de represión o autoritarismo, tratamos de dar un segundo paso, ahora a escala de las ciencias sociales en América Latina, como complemento del paso anterior y con el horizonte de avanzar en la consolidación de un área de estudios de los procesos emocionales al interior de la movilización social y el activismo.

\section{Presentación del Dossier}

La sección lente de aproximación abre con el artículo de Fabricio Teló, titulado "Campesinos, emociones y tentativas de resistencia armada a la dictadura empresarial-militar de Brasil.” El autor analiza la relación entre militantes de organizaciones armadas y campesinos en un contexto altamente represivo y de violencia estatal en plena dictadura militar brasileña. En base a un estudio de caso en el Estado de Rio de Janeiro, a partir del uso de historias de vida y la aplicación de entrevistas a sobrevivientes del periodo, el texto plantea cómo las emociones han interferido en los procesos de participación, desconexión y no participación de los campesinos en los intentos de formar grupos guerrilleros rurales. El escrito reconoce que las emociones son una importante herramienta analítica para comprender mejor las acciones colectivas y los compromisos de los actores implicados en situaciones de represión y violencia política.

A continuación, el artículo "Los programas sociales como mecanismos de "represión desapercibida” en Argentina (2007-2019). Un análisis desde las políticas de las sensibilidades”, escrito por Adrián Scribano y Angélica de Sena, problematiza la llamada politización de la sociedad argentina y la ampliación del campo de los derechos sociales, a partir del reconocimiento de su lado oscuro. La otra cara del proceso de politización argentino post2001, es un llamado de atención sobre los procesos de represión, silenciamiento y vulneración de derechos que le han acompañado. En base al análisis de datos primarios y secundarios, de naturaleza mixta, Scribano y de Sena muestran lo que podría parecer una cara amable de la represión, pero que viene a cuestionar profundamente la forma en que la definimos, al problematizar la relación entre políticas sociales y emocionalidades/sensibilidades en la politización y su contracara. El texto concluye que la "represión desapercibida" ha transformado a la represión física en un mecanismo reservado para momentos y actores muy específicos en la Argentina del presente.

De la represión explícita en el texto de Teló a la represión desapercibida de Scribano y de Sena, pasamos a procesos en los que el trabajo emocional de los actores colectivos implica un escenario de subversión emocional. El artículo de Carolina Tavano, intitulado "Nuestra única venganza es ser felices”: emociones, sentimientos y militancias de HIJOS en Espacios de memoria”, desde una perspectiva sociocultural y en base a un trabajo cualitativo narrativo, analiza los sentidos, emociones y sentimientos que se construyen entre los militantes de la agrupación H.I.J.O.S., a partir de la participación en el Espacio de memoria Ex ESMA, tanto en una dimensión endógena hacia la agrupación y sus integrantes, como también en relación a las autoridades del Gobierno 
Nacional, en un marco temporal de 12 años (2003-2015).Mediante el análisis de esta experiencia se dará cuenta de cómo sentimientos como la injusticia o la impunidad y emociones de rabia y dolor son procesados colectivamente a partir dela organización colectiva y transformados, desafiando las" reglas del sentir” sobre diferentes situaciones de represión compartidas, experimentados por miembros de la organización.

En un registro temporal diferente, Nicolás Ortiz R., a partir de la represión vivenciada en las jornadas de protesta estudiantiles en Chile, durante el año 2011,el artículo "Cacerolazo: emociones y memoria en el movimiento estudiantil 2011",analiza, a través de los relatos de 4 activistas, la masiva y espectacular lucha estudiantil de ese año desde el fenómeno del Cacerolazo y su relación con un trabajo sobre la memoria reciente. El artículo reflexiona respecto a la capacidad de enmarcar que tuvo la movilización con éste tipo de actuación, al utilizarlo como un ejercicio de irrupción de la memoria de la lucha civil contra la dictadura chilena. Por lo anterior, el cacerolazo se presenta como una forma de trabajo emocional que logra canalizar los sentimientos de temor e impotencia que surgen a partir de la violenta represión de la policía contra los activistas, permitiendo mantener la intensidad de la movilización en base a un estado emocional compartido. Por otro lado, el marco emocional del Cacerolazo logra resonar no sólo en activistas, sino que también en la población en general, quienes participan colectivamente en el ritual de protesta. El artículo termina exponiendo la relevancia que tiene la memoria en contextos contenciosos y su potencial para canalizar emociones y significados durante estos procesos.

Cierra la sección, en una suerte de contrapunto de los trabajos anteriores al no enfatizar al dimensión represiva ni autoritaria, el artículo "Acciones de participación social, identidad y emociones de estudiantes chilenos de una universidad privada Chile” de Villagrán et alt. El artículo a partir de una larga data de movilizaciones relacionadas con el acceso a la educación en la última década en Chile, que ha privilegiado el estudio de la educación pública y sus alumnos, pone su atención en el caso de universidades privadas. El trabajo analiza la relación entre las variables de emociones, identidades (colectivas y políticas) como las modalidades de participación en estudiantes de una universidad privada. En el estudio participaron 224 estudiantes de la carrera Psicología de una universidad privada chilena, con edades entre 19 y 33años. A partir de los distintos resultados originados por el cruce de las variables emocionales en la identidad y la participación, el artículo discuten las implicaciones de estos hallazgos y de las posibilidades y limitaciones que se presentan para los estudiantes universitarios de involucrarse y participar en el Movimiento Estudiantil desde una universidad privada.

El número se complementa con los textos de la sección Resultados de Investigación y las reseñas.

\section{Bibliografía}

Barker, C.(2001). Fear, Laughter and Collective Power: The Making of Solidarity at the Lenin Shipyard in Gdansk, Poland, August 1980. En, J.,Goodwin, J.M., James, y F., Polletta, (eds). Passionate Politics: Emotions and Social Movements. Chicago, United States: University of Chicago Press.

Lamb-Books, B. (2016). Angry Abolitionists and the Rhetoric of Slavery Moral Emotions in Social Movements. New York, United States: Palgrave Macmillan

Bolívar, I.J. (2006). Discursos emocionales y experiencias de la política: las FARC y las AUC en los proceso de negociación del conflicto (1998-2005). Bogotá, Colombia: Ediciones Uniandes.

Brym, R. J. y Araj, B. (2006). Suicide Bombing as Strategy and Interaction: The Case of the Second Intifada. Social Forces, 84(4): 1969-1986.

Della Porta, D.(1998). Las motivaciones individuales en las organizaciones políticas clandestinas. En P., Ibarra, y B. Tejerina (eds.) Los movimientos sociales. Transformaciones políticas y cambio cultural. Madrid, España: Editorial Trotta. 
Flam, H.(1998). Mosaic of Fear: Poland and East Germany before 1989. New York, United States: Columbia University Press.

(2005). Emotion's map: a research agenda. En H. Flam, y D. King (eds.). Emotions and Social Movement, (pp.19 - 40). London, England: Routledge.

Goodwin, J. y Pfaff, S. (2000). Emotion work in high-risk social movements: managing fear in the U.S. and East German civil rights movements”. En J. Goodwin, J. M, Jasper y F., Polletta. (eds), Passionate Politics: Emotions and Social Movements, (pp. 282-302). Chicago, United States: University of Chicago Press.

Gravante, T. y Poma, A. (2019). Introducción. En 'Dossier monográfico: 25 años de movimiento zapatista: desde el desencanto a la digna rabia’. Boletín Onteaiken. Programa de Estudios sobre Acción Colectiva y Conflicto Social, 14(27): i-iii.

Hochschild, A. (1975). The Sociology of Feeling and Emotion: Selected Possibilities. En Another Voice, Millman, M. y Moss Kanter (eds.). New York: Anchor.

(1979). Emotion Work, Feeling Rules, and Social Structure. American Journal of Sociology, 85, 551-75. (1983).The Managed Heart. Commercialization of Human Feeling. Berkeley, CA: University of California Press.

(2008). La mercantilización de la vida íntima. Apuntes de la casa y el trabajo. Buenos Aires: Katz.

Jasper, J.M. (1997). The Art Moral of Protest: Culture, Biography, and Creativity in Social Movements. Chicago, United States: University Chicago Press.

(2018). The Emotions of Protest. Chicago, United States: University of Chicago Press.

McAdam, D.(1986). Recruitment to High-Risk Activism: The Case of Freedom Summer. American Journal of Sociology,92 (1): 64-90.

Otero, S. (2016). Emociones y movimientos sociales: algunas claves útiles para estudiar el conflicto armado. Colombia Internacional (63): 174-187.

Poma, A. y Gravante, T. (2017a). Emociones, protesta y acción colectiva: estado del arte y avances. Aposta. Revista de Ciencias Sociales, 74: 32-62.

Poma, A., y Gravante, T.(2017b). Protest, Emotion and Change: An Analysis of Two Women's Collectives Fighting against Machismo in Oaxaca, Mexico. Interface, 9(1): 204-229.

(2018a). Emociones, identidad colectiva y estrategias en los conflictos socio-ambientales. Andamios. Revista de Investigación Social, 15(36): 287-309. doi: 10.29092/uacm.v15i36.611

(2018b). Manejo emocional y acción colectiva: las emociones en la arena de la lucha política. Estudio Sociológico, 36(108): 593-616. DOI: 10.24201/es.2018v36n108.1612.

Pupavac, V. (2004). War on the couch: the emotionology of the new international security paradigm. European Journal of Social Theory. 7(2): 149-170.

Whittier, N. (2001). Emotional Strategies: The Collective Reconstruction and Display of Oppositional Emotions in the Movement against Child Sexual Abuse. En Passionate Politics: Emotions and Social Movements, Goodwin, J., James, J. M. y Polletta, F. (eds), 233-250. Chicago: University of Chicago Press. 
Wood, E. J. (2003). Insurgent Collective Action and Civil War in El Salvador. Cambridge: Cambridge University Press.

Zeldin, Th. (1979). France 1848-1945: Politics and Anger. Oxford: Oxford University Press. 